\title{
Development of spin correlations in the geometrically frustrated triangular-lattice Heisenberg antiferromagnet $\mathrm{CuCrO}_{2}$
}

\author{
R. Kajimoto,, ,, 田 K. Tomiyasu, ${ }^{3}$ K. Nakajima, ${ }^{2}$ S. Ohira-Kawamura, ${ }^{2}$ Y. Inamura, ${ }^{2}$ and T. Okuda ${ }^{4}$ \\ ${ }^{1}$ Research Center for Neutron Science and Technology, CROSS, Tokai, Ibaraki 319-1106, Japan \\ ${ }^{2} J$-PARC Center, Japan Atomic Energy Agency, Tokai, Ibaraki 319-1195, Japan \\ ${ }^{3}$ Department of Physics, Tohoku University, Sendai 980-8578, Japan \\ ${ }^{4}$ Department of Nano-Structures and Advanced Materials, \\ Kagoshima University, Kagoshima 890-0065, Japan
}

(Dated: April 29, 2022)

\begin{abstract}
Magnetic excitations in the triangular-lattice Heisenberg antiferromagnet (TLHA) $\mathrm{CuCrO}_{2}$ were studied using single-crystal inelastic neutron scattering. A diffusive quasielastic component that persisted without developing a correlation length over a wide temperature range both below and above the ordering temperature was observed. Furthermore, characteristic momentum dependence was observed that was reproduced using minimum spin clusters. The robust spin clusters contrast with conventional magnetic ordering and may be universal in TLHAs.
\end{abstract}

PACS numbers: 75.25.-j, 75.40.Gb, 75.47.Lx

A two-dimensional (2D) triangular-lattice Heisenberg antiferromagnet (TLHA) is a typical and one of the simplest examples of geometrically frustrated antiferromagnets, in which a novel spin state originating from competing magnetic interactions and low dimensionality is expected. Although the study of this type of system originated with the resonating valence bonds theoretically predicted by Anderson more than four decades ago 1], the realization of this novel state was not confirmed experimentally until recently, and this system still remains of great interest in condensed matter physics. The spin liquid state was first confirmed in the organic $S=1 / 2$ systems, $\kappa$-(BEDT-TTF $)_{2} \mathrm{Cu}_{2}(\mathrm{CN})_{3}$ [2] and $\mathrm{EtMe}_{3} \mathrm{Sb}\left[\operatorname{Pd}(\mathrm{dmit})_{2}\right]_{2}[\underline{3}, 4]$, for which the quantum fluctuations of $S=1 / 2$ spins prevent antiferromagnetic spin ordering, even at zero temperature $(T)$. Furthermore, other novel spin states can be realized in larger $S$ systems with short-range spin correlations. For example, thermodynamic and powder neutron scattering studies of $\mathrm{NiGa}_{2} \mathrm{~S}_{4}$ revealed that this material shows a low- $T$ disordered state of $S=1$ spins with short-range correlations, and this behavior was interpreted as the formation of a spin liquid state [5]. Furthermore, an $S=3 / 2$ system, $\mathrm{NaCrO}_{2}$, for which the classical nature of the spins should dominate, shows an unconventional fluctuating crossover regime at finite $T$ below the spin transition temperature $T_{c} \sim 40 \mathrm{~K}[6]$. A powder neutron scattering study showed that the spin correlations induce diffuse quasielastic scattering in this $T$ region [7]. This spin fluctuation is speculated to be an evidence of excitations of $Z_{2}$ vortices [8 10], although its origin has not yet been identified.

The delafossite oxide $\mathrm{CuCrO}_{2}$ is a 2D TLHA. This compound is similar to the ordered rock-salt compound $\mathrm{NaCrO}_{2}$ in that the $S=3 / 2$ spins of the $\mathrm{Cr}^{3+}\left(3 \mathrm{~d}^{3}\right)$ ions form a $2 \mathrm{D}$ triangular lattice, and the $\mathrm{Cr}$ layers stack in a rhombohedral manner $(A B C A B C \cdots)$ [1]]. In this compound, 2D spin correlations begin to develop around the Curie-Weiss temperature $T_{\mathrm{CW}}=160-200 \mathrm{~K}$ [12 16]. Because of finite inter-layer couplings, three-dimensional (3D) ordering of a nearly $120^{\circ}$ structure occurs below $T_{N} \sim 24 \mathrm{~K}[16-18]$. However, the magnetic specific heat $\left(C_{\text {mag }}\right)$ exhibits a broad shoulder structure in addition to the sharp peak for the $3 \mathrm{D}$ spin ordering around $T_{N}$ 19], suggesting the existence of additional spin fluctuations. Although the sharp peak is suppressed by the substitution of the $\mathrm{Cu}^{+}\left(\mathrm{Cr}^{3+}\right)$ ion with a $\mathrm{Ag}^{+}\left(\mathrm{Al}^{3+}\right)$ ion reflecting the suppression of the $3 \mathrm{D}$ ordering, the broad shoulder structure remains, which together with the $T^{2}$ dependence of $C_{\text {mag }}$ at low $T$ suggests unconventional 2D low-energy spin fluctuations [14,20]. Concomitantly with these anomalies in $C_{\mathrm{mag}}$, diffuse quasielastic scattering similar to that observed in $\mathrm{NaCrO}_{2}$ is commonly observed via neutron scattering [21, 22], confirming the existence of dynamical 2D short-range spin correlations. However, because the neutron scattering studies of these $\mathrm{Cr}$ compounds have been limited to powder samples, detailed information, particularly regarding the spatial (momentum) distribution of the spin fluctuations, has not yet been obtained.

Accordingly, in this Letter, we report an inelastic neutron scattering (INS) study of a single crystal of $\mathrm{CuCrO}_{2}$ for detailed elucidation of the diffuse component in the magnetic excitation spectrum observed for powder samples. Close investigation revealed a unique $Q$ and $T$ dependence of the diffuse component. The origin of this correlation and its relationship to the development of spin correlations in TLHA are also discussed.

A $140 \mathrm{mg}$ single crystal of $\mathrm{CuCrO}_{2}$ was synthesized by a flux method. The crystal structure was rhombohedral (space group $R \overline{3} m$ ) with lattice constants of $a \sim 3.0 \AA$ and $c \sim 17 \AA$ in a hexagonal setting [14]. The INS measurement was performed using the ColdNeutron Disk-Chopper Spectrometer AMATERAS at JPARC [23]. The incident energy of the neutrons and the 

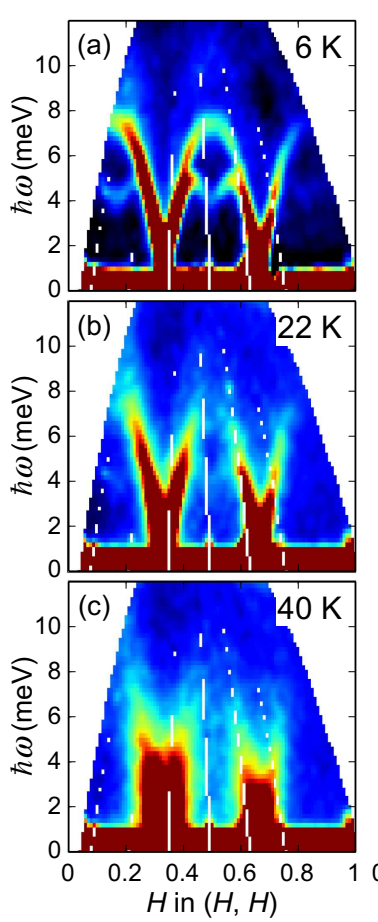
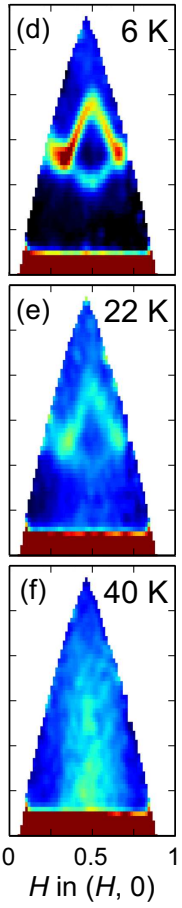
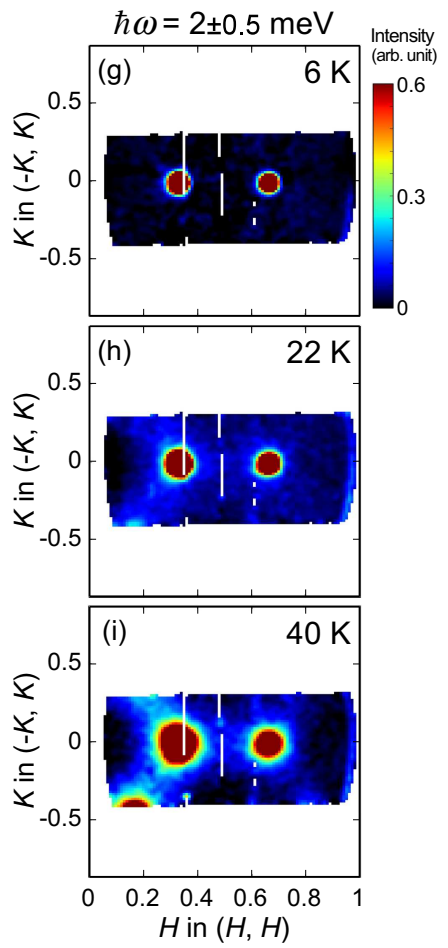
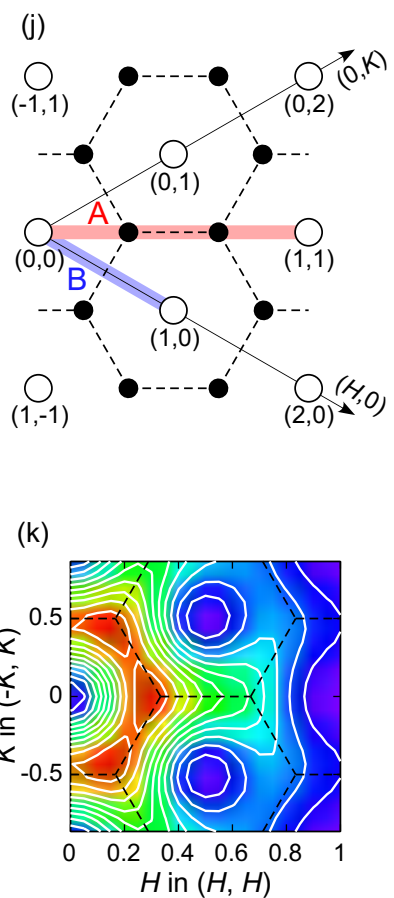

FIG. 1. (Color online) (a)-(i) Excitation spectra of $\mathrm{CuCrO}_{2}$ at 6,22 , and $40 \mathrm{~K}$. (a)-(c) The $Q$ - $\hbar \omega$ maps cut along $(H, H$ ) [line A in (j)] with widths of $K=0 \pm 0.04$ in $(-K, K)$. (d)-(f) The $Q$ - $\hbar \omega$ maps cut along $(H, 0)$ [line B in (j)] with widths of $K=0 \pm 0.02$ in $(-K, 2 K)$. (g)-(i) Maps on the $(H, H)-(-K, K)$ planes cut at $\hbar \omega=2 \mathrm{meV}$ with widths of $\pm 0.5 \mathrm{meV}$. (j) $2 \mathrm{D}$ reciprocal lattice of $\mathrm{CuCrO}_{2}$. Open circles, closed circles, and broken lines show $\Gamma$ points, $\mathrm{K}$ points, and Brillouin zone boundaries, respectively. A and B show the directions of the cuts of data (see text). (k) Neutron scattering intensity map calculated based on the trimer model. Broken lines show Brillouin zone boundaries.

energy resolution at elastic scattering were $15 \mathrm{meV}$ and $1 \mathrm{meV}$, respectively. All the data were analyzed using the software suite UTSUSEMI [24] to obtain the dynamical structure factor $S(\mathbf{Q}, \omega)$ as described in [21] ( $\mathbf{Q}$ and $\hbar \omega$ are the momentum and energy transfers, respectively). The crystal was initially aligned such that $\mathbf{c}$ was parallel to the incident neutron beam and the [110] direction was horizontal, and then rotated about the vertical axis by $40^{\circ}$. The $Q_{z}$ dependence of the data was ignored, because the magnetic excitations were quasi 2D 15, 25, whereas the $\mathbf{Q}$ dependence was represented by the $2 \mathrm{D}$ hexagonal reciprocal lattice $\left(Q_{x}, Q_{y}\right)=\left(H a^{*}, K a^{*}\right)$ with $a^{*}=4 \pi /(\sqrt{3} a)$ [Fig. 1(j)]. This configuration and analysis enabled mapping of the $2 \mathrm{D}$ reciprocal space on the $2 \mathrm{D}$ detector arrays of the instrument. For the data at the lowest $T=6 \mathrm{~K}$, the data for an empty aluminum cell filled with helium gas were subtracted to correct for the background due to scattering of the helium in the sample cell. For the measurement of the magnetic Bragg peak [Fig. 3(a)], the monochromating chopper was halted in the open position (diffraction mode). A preliminary result of the present study was reported in [26].

First, the magnetic Bragg peak was measured to define the value of $T_{N}$ for the sample. Figure 3(a) shows the $T$ dependence of the integrated intensity of the magnetic
Bragg peak $(1 / 3,1 / 3,0)$. From these data, the value for $T_{N}$ of the present sample was observed to be $24 \mathrm{~K}$, which is the temperature at which the intensity shows a clear increase with decreasing $T$.

Figures 1(a)-(c) show the excitation spectra at 6 , 22 , and $40 \mathrm{~K}$, which are cut along $(H, H)$ [line $\mathrm{A}$ in Fig. 1(j)]. At $6 \mathrm{~K}$, the spin-wave excitations dispersing from the magnetic Bragg peak positions (K points), $\mathbf{Q}=$ $(1 / 3,1 / 3)$ and $(2 / 3,2 / 3)$, were observed over $\sim 8 \mathrm{meV}$ [Fig. 1(a)], and the dispersion relation was consistent with previously reported results obtained at $2 \mathrm{~K}$ [15]. It should be noted that a continuum scattering with less intensity existed above the spin wave dispersion over $\sim 10 \mathrm{meV}$, which likely originated from two-magnon scattering [27]. As $T$ increased, the spectrum broadened [Figs. 1(b) and 1(c)]. Furthermore, a weak and diffusive signal filling the $Q-\hbar \omega$ region between the dispersions from $(1 / 3,1 / 3)$ and $(2 / 3,2 / 3)$ was detected. The appearance of the diffuse scattering can be distinguished more clearly when the excitation spectrum is cut along $(H, 0)$ [line B in Fig. 1(j)], as shown in Figs. 1(d)-(f). At 6 K, two branches of the spin wave excitations were observed in the region $\hbar \omega=4-8 \mathrm{meV}$ [Fig. 1(d)]. At $22 \mathrm{~K}$, the diffuse scattering appeared at approximately $H=0.5$, connecting the spin wave branches and $\hbar \omega=0 \mathrm{meV}$ 

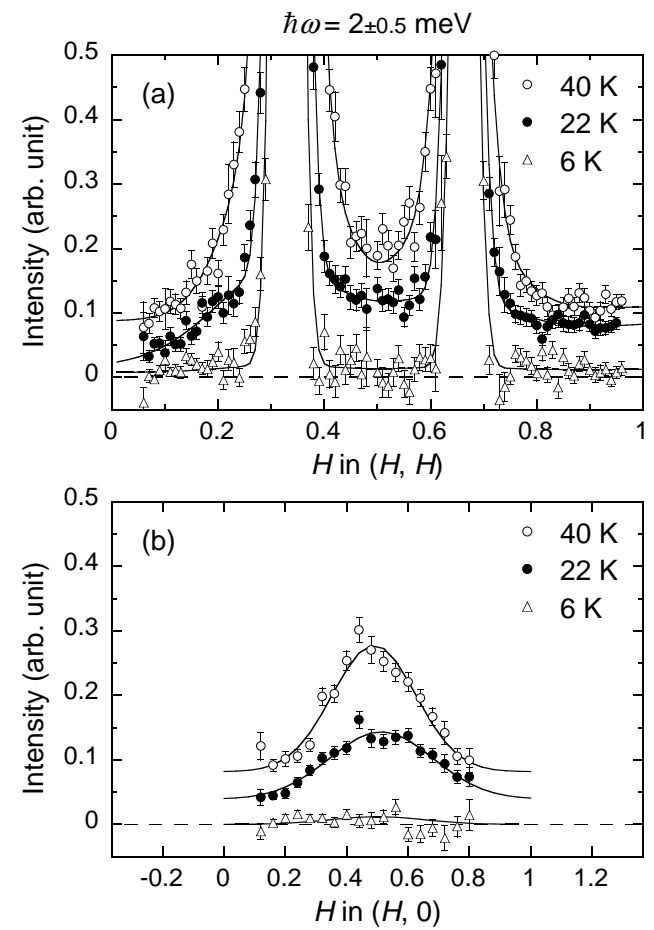

FIG. 2. Line profiles of the excitations at 6,22 , and $40 \mathrm{~K}$ cut at $\hbar \omega=2 \pm 0.5 \mathrm{meV}$ and sliced (a) along $(H, H)$ [line $\mathrm{A}$ in Fig. 1(j)] with a width of $K=0 \pm 0.02$ in $(-K, K)$ and (b) along $(H, 0)$ [line B in Fig. 1(j)] with a width of $K=0 \pm 0.02$ in $(-K, 2 K)$. Solid lines are fits to Gaussians as described in the text.

[Fig. 1(e)]. It should also be noted that, in this $T$ region slightly below $T_{N}$, the diffuse scattering coexisted with the spin wave branches. At $40 \mathrm{~K}$, the diffuse scattering became more intense, whereas the spin wave branches were severely damped [Figs. 1(c) and 1(f)].

To examine the $\mathbf{Q}$ dependence of the diffuse scattering, the data was cut at $\hbar \omega=2 \mathrm{meV}$. Figures 1(g)-(i) show the obtained spectra on the $(H, K)$ plane at 6,22 , and $40 \mathrm{~K}$. At $6 \mathrm{~K}$, two bright spots were observed at $(1 / 3,1 / 3)$ and $(2 / 3,2 / 3)$, which originated from the spin waves [Fig. 1(g)]. As $T$ increased to $22 \mathrm{~K}$, the diffuse scattering appeared as ridges connecting the spin-wave spots [Fig. 1(h)]. Additionally, the intensity of the diffuse scattering was pronounced as $T$ was further elevated to $40 \mathrm{~K}$ [Fig. 1(i)]. Notably, the intensity of the diffuse scattering decreased as $Q$ increased, which evidences that it is of the magnetic origin.

To further characterize the two components of the magnetic excitations, the $T$ dependence of their $Q$ profiles was investigated more quantitatively. Figure 2 shows the cuts of the spectra in Figs. 1(g)-(i) along $(H, H)$ and $(H, 0)$. At 22 and $40 \mathrm{~K}$, the profiles along $(H, H)$ consisted of broad peaks for the diffuse scattering around sharp peaks for the spin wave excitations at $H=1 / 3$ and $2 / 3$ [Fig. 2(a)]. The profiles along $(H, 0)$, on the other hand, exhibited broad single peaks at $H=0.5$
[Fig. 2(b)]. The intensity of the broad component decreased as $T$ decreased, and was rarely observed at $6 \mathrm{~K}$. Next, to parameterize the $T$ dependence, least-square fittings were performed as follows. For the profiles along $(H, H)$, the intensity $(I)$ was fit to a combination of two sharp Gaussians of the spin wave component, two broad Gaussians of the diffuse component, and the background $(B)$, as expressed by the following equation:

$I=|f(Q)|^{2} \sum_{H_{c}}\left[A_{s} e^{-\ln 2 \frac{\left(H-H_{c}\right)^{2}}{\kappa_{s}^{2}}}+A_{d} e^{-\ln 2 \frac{\left(H-H_{c}\right)^{2}}{\kappa_{d}^{2}}}\right]+B$.

Here, $A_{s}\left(A_{d}\right)$ and $\kappa_{s}\left(\kappa_{d}\right)$ are the amplitude and half width at half maximum (HWHM) of the spin wave component (the diffuse component), respectively. $H_{c}$ is the center of each peak $\left(H_{c} \sim 1 / 3\right.$ and $\left.2 / 3\right)$, and $f(Q)$ is the magnetic form factor $\left\langle j_{0}\right\rangle$ for $\mathrm{Cr}^{3+}[28]$. The profiles along $(H, 0)$ were fit to single broad Gaussians with the background. Linearly sloping and constant backgrounds were assumed for the profiles along $(H, H)$ and $(H, 0)$, respectively. The results of the fittings for the 6,22 , and $40 \mathrm{~K}$ data are shown as solid lines in Fig. 2.

Figures 3(b) and 3(c) compile the results of the $T$ dependence fittings of the integrated intensities and HWHM, respectively. The integrated intensity was corrected using the Bose factor $n(\omega, T)+1=$ $\left[\exp \left(\hbar \omega / k_{B} T\right)-1\right]^{-1}+1$. Because of the weakness of the diffuse components at 6 and $120 \mathrm{~K}$, the values at $30 \mathrm{~K}$ were used for the HWHMs along $(H, H)$ at 6 and $120 \mathrm{~K}$ and $(H, 0)$ at $6 \mathrm{~K}$. At $250 \mathrm{~K}$, the intensity of the excitation spectrum was too weak to distinguish the two components. The profile along $(H, H)$ was then fit to two Gaussians at $H=1 / 3$ and $2 / 3$. With decreasing $T$, the intensity of the spin wave component increased, and was nearly saturated below $T_{N}$. The diffuse component further increased with decreasing $T$, and then decreased after exhibiting a broad maximum at $T_{\text {peak }} \sim 35 \mathrm{~K}$. By studying a powder sample [21], it was confirmed that a finite amount of the diffuse component survives above $13 \mathrm{~K}$, but becomes negligible at $6 \mathrm{~K}$. With respect to the peak widths, the HWHM of the spin wave component monotonically decreased as $T$ decreased, indicating the development of the correlation length, although it did not diverge at $T_{N}$. On the other hand, that of the diffuse component exhibited no systematic $T$ dependence except for a slight increase.

One of the interesting features of the diffuse magnetic excitations is that they existed on the lines connecting the $\mathrm{K}$ points in the $2 \mathrm{D} Q$ space [Figs. I(h) and 1(i)]. Diffuse quasielastic or inelastic scatterings with characteristic $Q$ dependence have also been observed in several spinels in which the corner-shared tetrahedra of spins form 3D geometrically frustrated systems [29 33]. In these cases, the diffuse scatterings are interpreted as formation of uncorrelated spin-cluster-like quasiparticles. Thus, the experimental $\mathbf{Q}$ pattern revealed in the present 


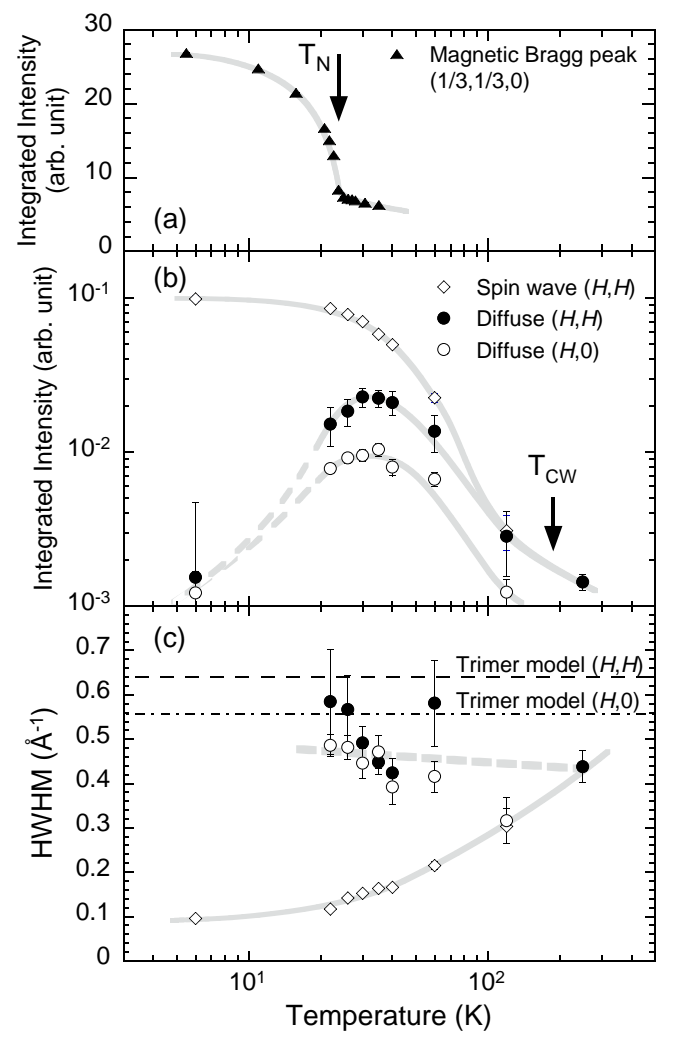

FIG. 3. (a) $T$ dependence of the integrated intensity of the magnetic Bragg peak $(1 / 3,1 / 3,0)$. $T$ dependences of $(b)$ the integrated intensities divided by the Bose factor and (c) peak widths (HWHM) of the excitation profiles at $\hbar \omega=2 \pm 0.5 \mathrm{meV}$ obtained by fitting the line profiles as shown in Fig. 2 In (c), HWHM is expressed in $\AA^{-1}$. Open squares, closed circles, and open circles show the data for the spin wave component along $(H, H)$, the diffuse component along $(H, H)$, and the diffuse component along $(H, 0)$, respectively. Gray lines are guides to the eye. In (c), the peak widths for the trimer model along $(H, H)$ and along $(H, 0)$ are shown as dashed and dashed-dotted lines, respectively.

study was compared to the two-body correlation function of a classical spin cluster model, as in [29, 32]. As a model, it was assumed that the neutrons are scattered by uncorrelated spin trimer units, in each of which three spins occupy the vertices of a minimum triangle corresponding to the constituent of the $120^{\circ}$ long-range antiferromagnetic order. Figure 1(k) shows the distribution of the neutron scattering intensities on the 2D $Q$ space based on this model, in which the magnetic form factor for $\mathrm{Cr}^{3+}$ was considered. The overall pattern of the observed neutron scattering intensities was well reproduced by this simple model. The widths of the intensity profiles along $(H, H)$ and $(H, 0)$, as shown in Fig. 3(c) by dashed and dashed-dotted lines, respectively, were obtained by cutting this calculated intensity pattern. Although the observed widths of the diffuse scattering profiles were smaller than the calculated values, indicating that the correlation length of the diffuse component was larger than the size of the trimer, the difference was not very significant.

Based on the trimer model, the following scenario is proposed for the development of the spin correlation in $\mathrm{CuCrO}_{2}$. Spins on a triangular unit of $\mathrm{Cr}$ ions begin to develop a $120^{\circ}$ correlation (trimer) below $\sim T_{\mathrm{CW}}$ that induces the diffuse scattering of the spin excitations. With decreasing $T$, a portion of these spins develop correlation lengths exhibiting 2D spin wave excitations. Simultaneously, the uncorrelated trimers increase their numbers and coexist with the matrix exhibiting the spin waves. After saturating around $T_{\text {peak }}$, the trimer correlation crosses over to the spin wave correlation. Although the uncorrelated trimers survive even below $T_{N}$, they finally disappear at $T=0$.

The broad maximum of the diffuse component around $T_{\text {peak }}$ should be related to the broad shoulder structure in $C_{\mathrm{mag}}$ around $T_{N}$ [19]. Similar broad shoulder structures in $C_{\mathrm{mag}}$ have been widely observed in other $S=3 / 2$ TLHAs and kagome-lattice antiferromagnets [5, 6, 14, 20, 34, 35], which have been attributed to the formation of spin singlets [35] or clusters [5]. The present result strongly suggests that the identity of these "singlets" or "clusters" is the spin trimers. Furthermore, the crossover behavior in the spin correlations in $\mathrm{CuCrO}_{2}$ is analogous to the unconventional crossover of spin fluctuations in $\mathrm{NaCrO}_{2}$ [6]. The similar decreases in the spin trimers for $\mathrm{CuCrO}_{2}$ should be the origin of the crossover phenomenon in $\mathrm{NaCrO}_{2}$. Thus, we believe that the crossover in the spin fluctuations accompanied by the uncorrelated trimers is a universal phenomenon in the development of the spin correlations in 2D TLHA.

Then, what is the origin of the trimer-like correlation in the spin fluctuations? One of the most fascinating possibilities is to relate the correlation to the formation of the $Z_{2}$-vortex [ 8 - 10]. Because the $Z_{2}$-vortex predicted in $2 \mathrm{D}$ TLHAs is a vortex of the chirality vectors defined by local $120^{\circ}$ structures, it results in essentially the same behavior as the trimer model when the correlation length is short. Recently, a theoretical study showed that the excitations of the $Z_{2}$-vortex should produce diffuse scattering connecting the $\mathrm{K}$ points [9]. Interestingly, the previously predicted $\mathbf{Q}$ pattern of the diffuse scattering looks very similar to those in Figs. 1(h) and 1(i). Furthermore, the $Z_{2}$-vortex shows a crossover to the spin wave correlations as $T$ decreases [9]. A recent electron spin resonance spectroscopy study of $A \mathrm{CrO}_{2}$ further suggested the existence of the $Z_{2}$-vortices at $T>T_{N}$ [36]. However, it remains unclear whether the $T$ independent correlation length of the spin trimers observed in the present study is consistent with the $Z_{2}$-vortices. If there is a $T$ region where the correlation length of the $Z_{2}$-vortex becomes considerably larger than that of the trimer but the spin wave correlation is not very developed, it would be possible to unambiguously distinguish the $Z_{2}$-vortex. Such a $T$ region may exist below $T_{\text {peak }}$, but a more detailed study 
with finer $T$ steps and higher statistics is required.

In conclusion, we performed an INS study of a single crystal of the $2 \mathrm{D}$ TLHA $\mathrm{CuCrO}_{2}$ to investigate the unconventional diffuse component in its magnetic excitations revealed during a previous study of a powder sample. We observed that the diffusive scattering shows a characteristic $Q$ pattern, which is consistent with scattering by spin clusters on minimum triangular-lattice units. The diffuse component crosses over to the spin wave component as $T$ decreases, but survives even below $T_{N}$ without developing its correlation length. The present results suggest that development of the spin correlation in TLHAs cannot be described solely by the divergence of the correlation length as in a conventional magnet, but is accompanied by a decrease in the robust spin clusters.

We thank T. Okubo, H. Kawamura, M. Itoh, Y. Kobayashi, K. Kakurai, and M. Arai for valuable discussions. The experiments on AMATERAS were performed with the approval of J-PARC (Proposal Nos. 2012A0113 and 2013A0087). This study was supported by JSPS KAKENHI Grant Nos. 25400378 and 26800174.

* E-mail: ryoichi.kajimoto@j-parc.jp

[1] P. W. Anderson, Mater. Res. Bull. 8, 153 (1973).

[2] Y. Shimizu, K. Miyagawa, K. Kanoda, M. Maesato, and G. Saito, Phys. Rev. Lett. 91, 107001 (2003).

[3] T. Itou, A. Oyamada, S. Maegawa, M. Tamura, and R. Kato, Phys. Rev. B 77, 104413 (2008).

[4] T. Itou, A. Oyamada, S. Maegawa, and R. Kato, Nat. Phys. 6, 673 (2010).

[5] S. Nakatsuji, Y. Nambu, H. Tonomura, O. Sakai, S. Jonas, C. Broholm, H Tsunetsugu, Y. Qiu, Y. Maeno, Science 309, 1967 (2005).

[6] A. Olariu, P. Mendels, F. Bert, B. G. Ueland, P. Schiffer, R. F. Berger, and R. J. Cava, Phys. Rev. Lett. 97, 167203 (2006).

[7] D. Hsieh, D. Qian, R. F. Berger, R. J. Cava, J. W. Lynn, Q. Huang, M. Z. Hasan, J. Phys. Chem. Solids 69, 3174 (2008).

[8] H. Kawamura and S. Miyashita, J. Phys. Soc. Jpn. 53, 4138 (1984).

[9] T. Okubo and H. Kawamura, J. Phys. Soc. Jpn. 79, 084706 (2010).

[10] H. Kawamura, J. Phys.: Conf. Ser. 320, 012002 (2011).

[11] S. Seki, Y. Onose, and Y. Tokura, Phys. Rev. Lett. 101, 067204 (2008).

[12] J. P. Doumerc, A. Wichainchai, A. Ammar, M. Pouchard, and P. Hagenmuller, Mater. Res. Bull. 21, 745 (1986).

[13] T. Okuda, N. Jufuku, S. Hidaka, and N. Terada, Phys. Rev. B 72, 144403 (2005).

[14] T. Okuda, T. Kishimoto, K. Uto, T. Hokazono, Y. Onose, Y. Tokura, R. Kajimoto, and M. Matsuda, J. Phys. Soc. Jpn. 78, 013604 (2009).

[15] M. Frontzek, J. T. Haraldsen, A. Podlesnyak, M. Matsuda, A. D. Christianson, R. S. Fishman, A. S. Sefat, Y. Qiu, J. R. D. Copley, S. Barilo, S. V. Shiryaev, and G.
Ehlers, Phys. Rev. B 84, 094448 (2011).

[16] M. Poienar, F. Damay, C. Martin, V. Hardy, A. Maignan, and G. André, Phys. Rev. B 79, 014412 (2009).

[17] H. Kadowaki, H. Kikuchi, and Y. Ajiro, J. Phys.: Condens. Matter 2, 4485 (1990).

[18] M. Soda, K. Kimura, T. Kimura, M. Matsuura, and K. Hirota, J. Phys. Soc. Jpn. 78, 124703 (2009).

[19] T. Okuda, Y. Beppu, Y. Fujii, T. Onoe, N. Terada, and S. Miyasaka, Phys. Rev. B 77, 134423 (2008).

[20] T. Okuda, K. Uto, S. Seki, Y. Onose, Y. Tokura, R. Kajimoto, and M. Matsuda, J. Phys. Soc. Jpn. 80, 014711 (2011).

[21] R. Kajimoto, K. Nakajima, S. Ohira-Kawamura, Y. Inamura, K. Kakurai, M. Arai, T. Hokazono, S. Oozono, and T. Okuda, J. Phys. Soc. Jpn. 79, 123705 (2010).

[22] R. Kajimoto, K. Nakajima, S. Ohira-Kawamura, Y. Inamura, K. Kakurai, T. Hokazono, S. Oozono, and T. Okuda, J. Phys. Soc. Jpn. 82, 054702 (2013).

[23] K. Nakajima, S. Ohira-Kawamura, T. Kikuchi, M. Nakamura, R. Kajimoto, Y. Inamura, N. Takahashi, K. Aizawa, K. Suzuya, K. Shibata, T. Nakatani, K. Soyama, R. Maruyama, H. Tanaka, W. Kambara, T. Iwahashi, Y. Itoh, T. Osakabe, S. Wakimoto, K. Kakurai, F. Maekawa, M. Harada, K. Oikawa, R. E. Lechner, F. Mezei, and M. Arai, J. Phys. Soc. Jpn. 80, SB028 (2011).

[24] Y. Inamura, K. Nakajima, R. Kajimoto, T. Nakatani, M. Arai, T. Otomo, J. Suzuki, J. Y. So, and J. G. Park, Proc. 19th Meet. Int. Collaboration of Advanced Neutron Sources, PSI-Proceedings 10-01 (2010); Y. Inamura, T. Nakatani, J. Suzuki, and T. Otomo, J. Phys. Soc. Jpn. 82, SA031 (2013).

[25] M. Poienar, F. Damay, C. Martin, J. Robert, and S. Petit, Phys. Rev. B 81, 104411 (2010).

[26] R. Kajimoto, K. Nakajima, S. Ohira-Kawamura, Y. Inamura, T. Okuda, and K. Tomiyasu, JPS Conf. Proc. 3, 014018 (2014).

[27] M. Mourigal, W. T. Fuhrman, A. L. Chernyshev, and M. E. Zhitomirsky, Phys. Rev. B 88, 094407 (2013).

[28] P. J. Brown, in International Tables for Crystallography Vol. C, edited by E. Prince (Wiley, Hoboken, NJ, 2004).

[29] S.-H. Lee, C. Broholm, W. Ratcliff, G. Gasparovic, Q. Huang, T. H. Kim, and S.-W. Cheong, Nature 418, 856 (2002).

[30] K. Kamazawa, S. Park, S.-H. Lee, T. J. Sato, and Y. Tsunoda, Phys. Rev. B 70, 024418 (2004).

[31] J.-H. Chung, M. Matsuda, S.-H. Lee, K. Kakurai, H. Ueda, T. J. Sato, H. Takagi, K.-P. Hong, and S. Park, Phys. Rev. Lett. 95, 247204 (2005).

[32] K. Tomiyasu, H. Suzuki, M. Toki, S. Itoh, M. Matsuura, N. Aso, and K. Yamada, Phys. Rev. Lett. 101, 177401 (2008).

[33] K. Tomiyasu, T. Yokobori, Y. Kousaka, R. I. Bewley, T. Guidi, T. Watanabe, J. Akimitsu, and K. Yamada, Phys. Rev. Lett. 110, 077205 (2013).

[34] H. Takatsu, H. Yoshizawa, S. Yonezawa, and Y. Maeno, Phys. Rev. B 79, 104424 (2009).

[35] A. P. Ramirez, B. Hessen, and M. Winklemann, Phys. Rev. Lett. 84, 2957 (2000).

[36] M. Hemmida, H.-A. Krug von Nidda, and A. Loidl, J. Phys. Soc. Jpn. 80, 053707 (2011). 